Journal of

Synchrotron

Radiation

ISSN 0909-0495

\title{
Progress in research into radiation damage in cryo-cooled macromolecular crystals
}

The problem of radiation damage to cryo-cooled macromolecular crystals in intense synchrotron beams has entered the mainstream of macromolecular crystallography (MX) during the last few years. A growing body of researchers worldwide are now carrying out systematic investigations into different aspects of this problem. The Fourth International Workshop on X-ray Radiation Damage to Biological Crystalline Samples was held on 7-8 March 2006 at SPring-8 in Japan and was attended by 80 researchers, the meeting being generously funded by SPring-8, Hyogo, Japan, and the Photon Factory in Tsukuba, Japan. The series of workshops on $X$-ray Damage to Biological Crystalline Samples aims to discuss and disseminate the latest research, often prior to publication, on the understanding, control, correction and possible use of radiation damage occurring during macromolecular crystallography experiments. Papers from the second (2001) and third (2003) workshops can be found in special issues of the Journal of Synchrotron Radiation (RD2, 2002; RD3, 2005). In this issue of the Journal of Synchrotron Radiation, there are 12 papers covering various aspects of current research into this area, including nine arising from the presentations made during the workshop.

It is now generally recognized that information deduced from three-dimensional biological structures can be compromised because of radiation-induced effects. In addition to the general degradation of diffraction properties associated with increasing dose, specific structural damage is observed to occur at $100 \mathrm{~K}$ in a well defined sequence, with the disulfide bond becoming disordered by breakage and delocalization, followed by the decarboxylation of aspartates and glutamates (Weik et al., 2000; Burmeister, 2000; Ravelli \& McSweeney, 2000). Metal binding proteins are partly reduced during X-ray exposure (Berglund et al., 2002; Yano et al., 2005), and active sites seem particularly susceptible to damage (Weik et al., 2000). These observations imply that an awareness of radiation damage effects is essential before any mechanistic conclusions are drawn from, for instance, the oxidation state of a metal atom in an active site. Increasingly, the processes of radiation damage are being used to enhance phase information and thereby provide more accurate starting maps. As the trend towards multidisciplinary biological research continues, it becomes important to consider how experience from other scientific fields, for instance spectroscopic tools and electron microscopy, may aid research in MX.

Systematic experiments on the characteristics of radiation damage over the last five years have resulted in the identification of a number of the important beam and crystal parameters governing its rate of progression and character (reviewed by Ravelli \& Garman, 2006). Research reports in this Journal of Synchrotron Radiation issue can be loosely grouped into five different areas: investigations into the general dependencies of radiation damage on various parameters, the effects of damage on phasing power and ways to correct for it, the use of optical and Raman spectroscopy to study aspects of damage, the study of heating effects induced by the beam in cryo-cooled crystals, and, lastly, experience in electron microscopy and how this might be applied to MX.

In this issue, Shimizu et al. (2007) report the analysis of various radiation damage indicators in lysozyme crystals as a function of incident photon energy, concluding that between $6.5 \mathrm{keV}$ and $33 \mathrm{keV}$ there is no observable energy dependence of radiation damage on dose. The possible correlation between the rate of radiation damage and the solvent accessibility of glutamates and aspartate residues in the halophilic enzyme malate dehydrogenase is explored by Fioravanti et al. (2007), who find there to be no such correlation. Borek et al. (2007) describe a range of diffraction experiments carried out on crystals of several different proteins to investigate the effects of cryogen temperature on various processing statistics and on the observed specific structural damage. 
Aspects of the impact of radiation damage on the phasing of macromolecular structures is addressed by three of the papers presented here: González (2007), who compares the relative phasing power of a SAD experiment with a two-wavelength MAD Se-Met experiment; Schiltz \& Bricogne (2007), who discuss different models for the description of site-specific damage; and Holton (2007), who reports the use of XANES to quantify the damage to selenium sites in proteins.

The use of various off-line and on-line spectroscopies (UV-visible and Raman) to explore several phenomena are described by three groups. Southworth-Davies \& Garman (2007) have used the $400 \mathrm{~nm}$ signature of the disulfide anion and an on-line microspectrophotometer to screen for putative MX scavengers, while Beitlich et al. (2007) investigate the effect

of radiation damage on the reduction rates of three hemecontaining proteins, again using on-line spectroscopy. Raman spectroscopy has been successfully used to track damage to a brominated DNA crystal by McGeehan et al. (2007), opening up new possibilities for on-line monitoring of radiation damage

Experimental results on the heating of a glass bead imaged with an infrared camera are described by Snell et al. (2007) and promise the possibility of further measurements so that detailed comparisons could be made with the theoretical calculations already available (Mhaisekar et al., 2005).

Electron microscopists have long struggled with the problem of the radiation damage caused by electron bombardment, and Massover (2007) outlines strategies used to minimize and circumvent its effects, pointing to some areas which may be worth further investigation for MX. One of these is in specimen preparation, and Ravelli et al. (2007) describe first attempts to embed a protein crystal in plastic using techniques developed for electron microscopy samples.

The various results of the work presented and the ensuing discussions during the workshop could be broadly divided into three categories: resolved issues, unresolved issues, and new issues. The papers collected here address some of these. The resolved issues were concluded to be:

(i) Dose/dose-rate effects, other than from crystal heating, seem to be small ( $<10 \%$ on intensity) up to a flux density of $10^{15}$ photons $\mathrm{s}^{-1} \mathrm{~mm}^{-2}$ (Sliz et al., 2003; Leiros et al., 2006; Owen et al., 2006).

(ii) Experiments using an infrared camera to measure the X-ray beam heating of small glass beads (Snell et al., 2007) have indicated that the previous extensive theoretical modelling of heating effects is likely to be reliable. These models, when used to simulate heating of spherical crystals in an X-ray beam, show that the temperature rise for the flux densities currently used on most beamlines is not likely to be larger than $15 \mathrm{~K}$ (Mhaisekar et al., 2005).

(iii) Helium cooling (15 K) versus nitrogen (100 K) does not seem likely to give the factor of around five improvement in radiation lifetime that would be necessary to justify both the technical complications and the cost, although it may be useful for special cases. (iv) The maximum dose limit of $2 \times 10^{7} \mathrm{~Gy}$, proposed by analogy with the observed dose to reduce diffraction to half the original intensity $\left(I_{\mathrm{o}}\right)\left(\langle I\rangle=0.5 I_{\mathrm{o}}\right)$ in electron microscopy (Henderson, 1990), has been verified experimentally and seems to have general applicability. A maximum dose limit for macromolecular crystallography of $3 \times 10^{7} \mathrm{~Gy}$ has been proposed $\left(\langle I\rangle=0.7 I_{\mathrm{o}}\right)$ after which the biological information may be compromised (Owen et al., 2006).

Unresolved issues were identified as:

(i) The nature of any energy dependence of the rate of radiation damage; recent experimental results indicate no dependence (Weiss et al., 2005; Shimizu et al., 2007) in broad agreement with theoretical predictions (Arndt, 1984; Murray et al., 2004).

(ii) The identity of the gas(es) which escape the exposed crystal after warming. Radiation chemistry models seem to suggest that it might be hydrogen, but it may also be $\mathrm{CO}_{2}$. Experimental verification of this for $\mathrm{MX}$ at $100 \mathrm{~K}$ is still required.

(iii) The mechanisms of scavenger action. There is a need to understand the radiation chemistry involved in radioprotection and scavenging; for instance, its $\mathrm{pH}$ dependence, so that eventually rational design of scavengers will be possible and will feed into experimental design.

(iv) There is a need to provide (more) user-friendly software that takes radiation damage into account both for data collection and structure determination.

(v) What is the pathway of energy dissipation after photoabsorption by a (heavy) atom above the absorption edge (compared with below the edge)?

(vi) There is a pivotal need to understand the biological implications of radiation damage on structures, particularly to metal centres which are damaged very fast (redox), and active sites which appear particularly sensitive. It would be useful to see the introduction of the possibility to flag radiation artefacts in the Protein Data Bank.

Several new issues had emerged since the last Radiation Damage Workshop:

(i) Have we properly correlated the experience available from X-ray imaging, X-ray crystallography, and electron microscopy and diffraction in terms of the limiting resolution, dose deposited and specimen type?

(ii) There is a need to understand the nature of UV damage compared with X-ray damage, especially since it has been demonstrated that UV light can be used for crystal centring (Nanao \& Ravelli, 2006; Vernede et al., 2006).

(iii) Better methods for dealing with non-isomorphism (both caused by unit-cell changes and specific structural changes, including occupancy decrease of heavier atoms) are required.

A repeated theme from various contributors during the Fourth Workshop was that there is an absolute requirement to make a careful estimate of dose [e.g. using RADDOSE (Murray et al., 2004)] whenever possible. We feel that this information is an important consideration for experimental design, and should, wherever practical, be provided by the 
synchrotron beamline control software. In practice this means it is necessary to (i) measure and record the integrated in-line beam counts for each image to allow proper quantitative relative comparison between data taken on the same day to be made and, if the beam characteristics (size, profile, flux) and crystal size are known, the dose can be calculated and measurements compared with those on other beamlines and facilities; (ii) retain this information right through to scaling of structure factors.

It was concluded that these international workshops provide a valuable stimulus for new research and exchange of information, so it is proposed to hold the fifth workshop in Europe in 2008.

In establishing the stimulating programme we benefited from the help and advice of the other organisers (Colin Nave, Raimond Ravelli, Gerd Rosenbaum) and the local organisers Masaki Yamamoto and Soichi Wakatsuki, who also took the initiative in obtaining funding for the workshop. In addition, Raimond Ravelli provided helpful comments on this short report.

\section{References}

Arndt, U. (1984). J. Appl. Cryst. 17, 118-119.

Beitlich, T., Kühnel, K., Schulze-Briese, C., Shoeman, R. L. \& Schlichting, I. (2007). J. Synchrotron Rad. 14, 11-23.

Berglund, G. I., Carlsson, G. H., Smith, A. T., Szoke, H., Henriksen, A. \& Hajdu, J. (2002). Nature (London), 417, 463-468.

Borek, D., Ginell, S. L., Cymborowski, M., Minor, W. \& Otwinowski, Z. (2007). J. Synchrotron Rad. 14, 24-33.

Burmeister, W. P. (2000). Acta Cryst. D56, 328-341.

Fioravanti, E., Vellieux, F. M. D., Amara, P., Madern, D. \& Weik, M. (2007). J. Synchrotron Rad. 14, 84-91.

González, A. (2007). J. Synchrotron Rad. 14, 43-50.

Henderson, R. (1990). Proc. R. Soc. Lond. Ser. B, 241, 6-8.
Holton, J. M. (2007). J. Synchrotron Rad. 14, 51-72.

Leiros, H. K., Timmins, J., Ravelli, R. B. G. \& McSweeney, S. M. (2006). Acta Cryst. D62, 125-132.

Massover, W. H. (2007). J. Synchrotron Rad. 14, 116-127.

McGeehan, J. E., Carpentier, P., Royant, A., Bourgeois, D. \& Ravelli, R. B. G. (2007). J. Synchrotron Rad. 14, 99-108.

Mhaisekar, A., Kazmierczak, M. \& Banerjee, R. (2005). J. Synchrotron Rad. 12, 318-328.

Murray, J. W., Garman, E. F. \& Ravelli, R. B. G. (2004). J. Appl. Cryst. 37, 513-522.

Nanao, M. H. \& Ravelli, R. B. G. (2006). Structure, 14, 791-800.

Owen, R. L., Rudino-Pinera, E. \& Garman, E. F. (2006). Proc. Natl. Acad. Sci. USA, 103, 4912-4917.

Ravelli, R. B. G. \& Garman, E. F. (2006). Curr. Opin. Struct. Biol. 16, 624-629.

Ravelli, R. B. G., Haselmann-Weiss, U., McGeehan, J. E., McCarthy, A. A., Marquez, J. A., Antony, C., Frangakis, A. S. \& Stranzl, G. (2007). J. Synchrotron Rad. 14, 128-132.

Ravelli, R. B. G. \& McSweeney, S. M. (2000). Structure, 8, 315-328.

RD2 (2002). J. Synchrotron Rad. 9, 327-382.

RD3 (2005). J. Synchrotron Rad. 12, 257-328.

Schiltz, M. \& Bricogne, G. (2007). J. Synchrotron Rad. 14, 34-42.

Shimizu, N., Hirata, K., Hasegawa, K., Ueno, G. \& Yamamoto, M. (2007). J. Synchrotron Rad. 14, 4-10.

Sliz, P., Harrison, S. C. \& Rosenbaum, G. (2003). Structure, 11, 13-19.

Snell, E. H., Bellamy, H. D., Rosenbaum, G. \& van der Woerd, M. J. (2007). J. Synchrotron Rad. 14, 109-115.

Southworth-Davies, R. J. \& Garman, E. F. (2007). J. Synchrotron Rad. 14, 73-83.

Vernede, X., Lavault, B., Ohana, J., Nurizzo, D., Joly, J., Jacquamet, L., Felisaz, F., Cipriani, F. \& Bourgeois, D. (2006). Acta Cryst. D62, 253-261.

Weik, M., Ravelli, R. B., Kryger, G., McSweeney, S., Raves, M. L., Harel, M., Gros, P., Silman, I., Kroon, J. \& Sussman, J. L. (2000). Proc. Natl. Acad. Sci. USA, 97, 623-628.

Weiss, M. S., Panjikar, S., Mueller-Dieckmann, C. \& Tucker, P. A. (2005). J. Synchrotron Rad. 12, 304-309.

Yano, J., Kern, J., Irrgang, K.-D., Latimer, M. J., Bergmann, U., Glatzel, P., Pushkar, Y., Biesiadka, J., Loll, B., Sauer, K., Messinger, J., Zouni, A. \& Yachandra, V. K. (2005). Proc. Natl. Acad. Sci. USA, 102, 12047-12052. 